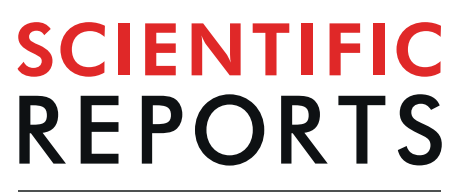

natureresearch

\title{
Autonomic adaptations mediate the effect of hydration on brain functioning and mood: Evidence from two randomized controlled trials
}

\author{
Hayley A. Young ${ }^{1 *}$, Alecia Cousins $\mathbb{1 D}^{1}$, Stephen Johnston $\mathbb{1}^{1}{ }^{1}$, John M. Fletcher ${ }^{2}$ \& \\ David Benton ${ }^{1}$
}

Dehydration (water loss $>\mathbf{2 . 0 \%}$ of body weight) has significant negative effects on physical and mental performance. In two studies the effects of minor hypo-hydration (water loss $<1.0 \%$ of body weight) on CNS function, mood and cardiovascular functioning were measured. Study 1: On two mornings twelve male participants were exposed to a temperature of $30^{\circ} \mathrm{C}$ for four hours and either did or did not drink two $150 \mathrm{ml}$ glasses of water during that time. Study 2: Fifty-six ( $25 \mathrm{M})$ individuals were exposed to the same $30^{\circ} \mathrm{C}$ environment and randomly allocated to either drink $(2 \times 150 \mathrm{ml})$ or not drink. When not given water $0.59 \%$ (Study 1 ) and $0.55 \%$ (Study 2) bodyweight was lost. Participant's heart rate variability (HRV) was measured, and they rated their thirst and mood. In study 1 , participants participated in an fMRI protocol during which they completed a modified version of the Paced Auditory Serial Addition Test (PASAT), at the end of which they rated its difficulty. Decreases in fMRI BOLD activity in the orbito-frontal cortex, ventral cingulate gyrus, dorsal cingulate cortex, hypothalamus, amygdala, right striatum, post-central gyrus and superior parietal cortex were observed when participants were hypo-hydrated. These deactivations were associated with reduced HRV, greater perceived effort, and more anxiety. In study 2 declines in HRV were found to mediate the effect of hypo-hydration on ratings of anxiety. These data are discussed in relation to a model that describes how autonomic regulatory and interoceptive processes may contribute to the affective consequences of minor hypo-hydration.

It has been assumed that small variations in hydration status, in what might be described as the normal day to day range (water loss $<1.0 \%$ of body weight), have no significant influence on higher mental functions such as cognition and $\operatorname{mood}^{1}$. Indeed, where dose - response relationships have been assessed, the largest psychological effects appear to occur with moderate levels of dehydration (water loss 2.0-5.0\% of body mass), although findings may depend on the method used to induce dehydration ${ }^{2}$. In healthy individuals such large changes in hydration status are rare and are associated with water deprivation and prolonged exposure to hot environmental temperatures and/or exercise. Therefore, the relevance of such findings to the majority of the population is unclear. It is argued that thirst and homoeostatic mechanisms keep the hydration status of those with access to water and living in temperate environments within a narrow range. However, it is plausible that even when fluid balance is successfully maintained, certain counter-regulatory mechanisms may themselves have psychological consequences - this possibility is yet to be explored. For the first time the present studies report that with as little as a $0.6 \%$ decrease in body mass, changes in cardiovascular and autonomic functioning (heart rate variability) negatively influence mood. These data highlight novel mechanisms that may contribute to the affective consequences of minor hypo-hydration.

The adverse consequences of dehydration (water loss $>2.0 \%$ of body mass) have been well described and include declines in physical and mental performance, and increased blood pressure and heart rate ${ }^{3}$. Questioning 
recieved wisdom we recently reported that participants exposed to a temperature of $30^{\circ} \mathrm{C}$ for four hours lost less than $1 \%$ of their body mass but experienced a significant decline in memory and focused attention; effects that were reduced by drinking water ${ }^{4,5}$. In an experimental protocol that tested performance on a simulated driving task, hypo-hydration (loss of $1.1 \%$ body mass) caused increased errors and changes in EEG alpha and theta waves indicative of drowsiness ${ }^{6}$. Interestingly, the most commonly observed consequences of minor hypo-hydration may be subjective ${ }^{2,7,8}$. For example, in the study by Benton, et al. ${ }^{4}$, an increase in thirst was associated with a decline in subjective energy and increased anxiety and depression. Therefore, given that fluid balance is highly regulated ${ }^{9}$, there is a need to understand the mechanisms underpinning these psychological consequences of minor hypo-hydration.

The physiological regulation of fluid balance is relatively well understood ${ }^{10}$, several forebrain and brainstem circuitries interact with peripheral neural and humoral signals to collaboratively maintain the osmolality and volume of fluids. One counter-regulatory mechanism involves changes in cardiovascular and autonomic functioning: hypo-hydration induces cardiovascular $\operatorname{strain}^{3}$, for every $1 \%$ decrease in body mass during exercise there is an increase in heart rate of 3.29 beats per $\min (\mathrm{bpm})^{11}$. Dehydration decreases the volume of blood (absolute hypovolemia), reducing cardiac output, and necessitating an increase in heart rate to maintain blood pressure. When there is a simultaneous elevation in skin temperature blood vessels are dilated resulting in relative hypovolemia; an inadequate distribution of blood volume between the periphery and core. These counter-regulatory changes, while part of normal body fluid homeostasis, may alter psychological functioning.

For example, fluid consumption has been shown to facilitate peripheral and cerebral perfusion when exposed to physiological ${ }^{12}$, orthostatic ${ }^{13}$ or psychological ${ }^{14}$ stress. Even in the absence of hypo-hydration, water ingestion is followed by an increase in cardiac vagal control ${ }^{15}$; an effect thought to counteract the pressor effects of sympathetic activation. Interestingly, neuroimaging studies that have attempted to understand our experience of hypo-hydration and thirst find changes in regions of the brain often associated with autonomic and cardiac control $^{16,17}$; that is regions comprising the central autonomic network ${ }^{18}$. For example, Farrell, et al. ${ }^{19}$ observed that a state of osmotic thirst (induced with hypertonic infusions) was related to changes in blood flow to the somatosensory and motor cortices, prefrontal cortex, anterior mid cingulate cortex, and superior temporal gyrus.

According to the neuro-visceral integration model of emotion regulation ${ }^{18}$, the central autonomic network mediating sympathetic and parasympathetic control include regions of the limbic forebrain such as the subgenual and pregenual cingulate, amygdala/ventral striatum and medial prefrontal corte ${ }^{20}$. Essentially the prefrontal cortex exerts inhibitory GABAergic control over the limbic regions of the brain that ultimately control heart rate ${ }^{20,21}$. This implies that in the presence of a physiological or psychological stressor that necessitates an increase in heart rate, there will be an associated decline in prefrontal neural activity. The relevance is that individual differences in vagal tone (measured using heart rate variability (HRV)) predict mood and cognitive performance, especially in tasks associated with the prefrontal and cingulate cortices ${ }^{2-25}$. Thus, it is plausible that cardiovascular and autonomic adaptations, may explain the affective consequences of minor hypo-hydration.

Although neuroimaging studies have attempted to understand our experience of thirst while at rest, only one study has considered the effect of hydration on brain functioning during a cognitive task. Kempton et al. ${ }^{26}$ studied the effects of fluid restriction during a thermal exercise protocol on fMRI BOLD responses to the Tower of London task. Participants lost $1.64 \%$ bodyweight and heart rate was about $20 \mathrm{bpm}$ higher during dehydration. There were no effects on two subjective rating of sedation although this may be explained by the confounding effects of physical activity. Similarly, performance on the task was not altered, participants had a greater BOLD response in the fronto-parietal cortex when dehydrated; an effect interpreted as reflecting a need for a higher level of neuronal activity to achieve the same performance level. Kempton et al. ${ }^{26} \mathrm{fMRI}$ analysis used a region-of interest approach which specifically examined task-related regions of activation. Therefore, it remains undetermined whether there were effects on brain regions linked with autonomic and cardiac control. In addition, although differences in the degree of bodyweight lost and core temperature were correlated with differences in neural activity, heart rate was not. Therefore, the possibility that alterations in cardiovascular or autonomic functioning mediate the association between hydration and psychological/affective functioning remains unexplored.

The aim of the present two studies was to determine whether cardiovascular and autonomic adaptations, may explain the affective consequences of minor hypo-hydration. Study one reports, for the first time, that change in blood flow to particular neural systems, as indicated by fMRI, can be observed with as little as a $0.6 \%$ decrease in body mass. It was shown that hydration deficits resulted in changed activity in the blood flow to the ventral cingulate gyrus (vCG) and medial orbito-frontal cortex (mOFC) that reflected autonomic adaptations to the physiological challenge of hypo-hydration (changes in HRV). In addition, it was observed that these adaptations correlated with changes in affect. Study two extends these novel findings showing that mild hypo-hydration reduced HRV that in turn mediated effects on mood.

\section{Statistical Analysis}

Hydration indices (Study 1 and Study 2). Fluid loss due to perspiration and breathing (hereafter referred to as perspiration rate) was estimated as the percentage change in body mass from baseline to the end of the session prior to urination (Fig. 1). Total body mass lost, including urination, was calculated as total percentage change in body weight. For body temperature and osmolality, change scores were calculated (end of the morning minus baseline). Similarly, thirst change scores were calculated (end of morning minus thirst following the standard breakfast).

Heart rate variability (Study 1 and Study 2). Interbeat (RR) intervals were recorded at rest for five minutes at the start of the procedure and again at the end. Interbeat interval data were analysed using Kubios HRV Analysis Software 2.0 27 (The Biomedical Signal and Medical Imaging Analysis Group, Department of Applied Physics, University of Kuopio, Finland). Data were visually inspected for artefacts caused by ectopic beats, poor 


\begin{tabular}{|c|c|c|c|}
\hline TIME & TASK & \multirow{9}{*}{$\begin{array}{l}\text { Fluid loss due } \\
\text { to perspiration } \\
\text { and breathing. }\end{array}$} & \multirow{9}{*}{$\begin{array}{l}\text { Total amount } \\
\text { of weight } \\
\text { lost. }\end{array}$} \\
\hline 8.30 & $\begin{array}{l}\text { - Osmolality } \\
\text { - Body temperature } \\
\text { - Pre-breakfast weight } \\
\text { - Pre-breakfast thirst }\end{array}$ & & \\
\hline 8.45 & $\begin{array}{l}\text { - Standard breakfast } \\
\text { - Post breakfast weight } \\
\text { - Post breakfast thirst }\end{array}$ & & \\
\hline 9.00 & $\begin{array}{l}\text { - Mood } \\
\text { - Heart rate variability }\end{array}$ & & \\
\hline 10.00 & $\begin{array}{l}\text { - Rest } \\
\text { - Drinks were provided at } \\
10.10 \text { and } 12.10\end{array}$ & & \\
\hline 12.40 & - Mood & & \\
\hline 12.50 & $\begin{array}{l}\text { - Body temperature } \\
\text { - Thirst } \\
\text { - Weight before urination }\end{array}$ & & \\
\hline 13.00 & $\begin{array}{l}\text { - Osmolality } \\
\text { - Weight after urination } \\
\text { - Heart rate variability }\end{array}$ & & \\
\hline 13.15 & - MRI procedure & & \\
\hline
\end{tabular}

Figure 1. The experimental procedure.

conductivity etc. A very low correction threshold was chosen for artefact correction ( 0.45 from local average) so not to distort natural variability. Less than $1 \%$ of beats were identified as artefacts. Time domain HRV indices included mean R-R interval (a measure of basic heart rate), the standard deviation of normal to normal R-R inter$\operatorname{val}(\mathrm{SDNN})$ (measures total variability in the series) and the root mean square of the standard deviation (RMSSD) (a measure of parasympathetic nervous system activity). To determine the effect of hydration, change scores were calculated (end of the morning minus baseline) for each index.

Subjective ratings (Study 1 and Study 2). For ratings of anxiety, depression and energy change scores were calculated (end of morning minus mood following the standard breakfast).

fMRI analysis (Study 1). BOLD sensitive echo planar images were collected as participants performed the arithmetic task $(\mathrm{TE}=30 \mathrm{~ms}, \mathrm{TR}=3000 \mathrm{~ms}$, flip angle $=90$, axial plane, interleaved acquisition, voxels $=3 \mathrm{~mm}$ $3 \mathrm{~mm} 3 \mathrm{~mm}$, slices $=44)$. In each of the three separate runs a total of 135 volumes were collected. Additionally a high-resolution magnetization-prepared rapid acquisition with gradient echo (MPRAGE) 3D volume image was acquired for each participant $(\mathrm{TE}=4.82 \mathrm{~ms}, \mathrm{TR}=2500 \mathrm{~ms}$, flip angle $=7$, sagittal plane, $1 \mathrm{~mm} 3$ isotropic voxels, 179 slices).

Analyses were conducted using BrainVoyager QX $2.8^{28}$ (Brain Innovations B.V., the Netherlands). Data were pre-processed as follows: 3D motion correction ( 6 degrees of freedom) with sinc interpolation to account for participant head movement during the scanning procedure, linear trend removal and temporal high pass filtering (low cutoff: 3 cycles per run), and spatial smoothing using a $6 \mathrm{~mm}$ Gaussian FWHM filter. Data were normalised to the standard Talairach space ${ }^{29}$ using sinc interpolation prior to group analysis. The group analysis involved estimating task-correlated activity using a general linear model (GLM) approach. The predictors for the GLM were created by convolving the timecourse of the stimulation periods (arithmetic operation) with a canonical HRF response. Each participants beta estimates, obtained via the GLM, were then input into a voxel wise second level random effects analysis. The threshold set for statistical significant was a voxel-wise $\mathrm{p}<0.05$, with an additional multiple comparison correction applied via cluster level thresholding using the Monte Carlo simulation tool implemented in BrainVoyager QX 2.8 (also with a statistical acceptance threshold of $\mathrm{p}<0.05$ ).

Arterial spin labelling (ASL) analysis (Study 1). For each participant an arterial spin labelling (ASL) sequence was run after the functional imaging experiment had been completed. The ASL sequence parameters were $\mathrm{TR}=2500 \mathrm{~ms}, \mathrm{TE}=12 \mathrm{~ms}$, in-plane resolution $=4 \mathrm{~mm} \times 4 \mathrm{~mm}, 90$ averages for each label and control pair, slice thickness $=8 \mathrm{~mm}$ with a $2 \mathrm{~mm}$ gap, 9 slices were collected. The labelling time was $700 \mathrm{~ms}$ and the post-labelling time was $1800 \mathrm{~ms}$. The labelling plane was placed $18.8 \mathrm{~mm}$ inferior to the bottom slice. Scans were 


\begin{tabular}{|c|c|c|c|c|}
\hline & \multicolumn{2}{|l|}{ Study 1} & \multicolumn{2}{|l|}{ Study 2} \\
\hline & No Water & Water & No Water & Water \\
\hline Weight lost before urination (\%) & $-0.40(0.01)^{* *}$ & $-0.01(0.03) * *$ & $-0.32(0.04)^{* *}$ & $-0.08(0.04 * *$ \\
\hline Weight lost after urination (\%) & $-0.59(0.03) * *$ & $-0.26(0.05) * *$ & $-0.54(0.05)^{*}$ & $-0.36(0.05)^{*}$ \\
\hline$\Delta$ Osmolality $(\mathrm{mOsm} / \mathrm{kg})$ & $219.77(57.50)^{* *}$ & $-190.00(55.47)^{* *}$ & $116.88(33.66) *$ & $-9.03(33.66)^{*}$ \\
\hline$\Delta$ Temperature $\left({ }^{\circ} \mathrm{C}\right)$ & $0.75(0.11)$ & $0.50(0.19)$ & $0.32(0.07)$ & $0.32(0.07)$ \\
\hline$\Delta$ Thirst (VAS) & $24.40(4.64)$ & $12.30(5.92)$ & $18.82(6.62)$ & $5.96(6.62)$ \\
\hline$\Delta \mathrm{R}-\mathrm{R}$ interval & $17.75(19.47)^{*}$ & $88.42(31.90)^{*}$ & $20.90(27.44) * *$ & $160.94(27.44) * *$ \\
\hline$\Delta \mathrm{SD}$ of R-R interval & $12.59(10.43)^{*}$ & $-12.80(5.68)^{*}$ & $-5.34(4.38)^{*}$ & $8.77(4.38) *$ \\
\hline$\Delta$ RMSSD & $3.87(6.96)^{*}$ & $13.38(5.89) *$ & $-0.67(4.44)^{*}$ & $13.21(4.44) *$ \\
\hline
\end{tabular}

Table 1. The effect of drinking compared with not drinking on hydration indices and heart rate variability. Data are mean (s.e.) for change scores across each morning (end of the morning minus baseline) for study 1 and study 2. In both studies, compared to when they drunk, when participants did not drink they lost more weight both before and after urination and their osmolality increased. They had a significantly greater increase in HRV (RR interval, SDNN, RMSDD) when they drunk compared to when they did not drink. $\Delta$ Change across the morning, *Water vs. No water significant at $\mathrm{p}<0.05$, **Water vs. No water significant at $\mathrm{p}<0.001$. RR, interbeat interval, SDNN, standard deviation of normal to normal R-R interval, RMSSD, root mean square of the standard deviation.

planned such that the ASL were in an AC-PC orientation, with the topmost scan aligned with the most superior aspect of the participant's brain. ASL data were 3D motion corrected and normalised to Talairach space. Relative perfusion values were obtained for each participant and condition using the ASL toolbox implemented in BrainVoyager QX 2.8. Significant differences between dehydrated and water conditions were assessed using paired t-test of the relative perfusion values.

\section{Results: Study 1}

Verification of the hydration paradigm. The effect of drinking on percentage total weight loss, perspiration rate and changes in osmolality, thirst and body temperature were analysed using RMANOVA. As expected when participants did not drink water they lost more weight both before $(\mathrm{F}(1,11)=214.853, \mathrm{p}<0.0001$, $\left.\eta_{\mathrm{p}}{ }^{2}=0.951\right)$ and after $\left(\mathrm{F}(1,11)=34.777, \mathrm{p}<0.0001, \eta_{\mathrm{p}}{ }^{2}=0.760\right)$ urination; in total an average of $0.6 \%$ of their body mass (Table 1). Hypo-hydration resulted in a significant increase in urine osmolality $(\mathrm{F}(1,11)=23.89$, $\left.\mathrm{p}<0.001, \eta_{\mathrm{p}}{ }^{2}=0.749\right)$ and participants tended to be more thirsty when they did not drink $(\mathrm{F}(1,11)=4.178$, $\left.\mathrm{p}<0.07, \eta_{\mathrm{p}}{ }^{2}=0.317\right)$. There was no difference in participants body temperature depending on whether or not they consumed water $\left(\mathrm{F}(1,11)=1.887, \mathrm{p}=0.197, \eta_{\mathrm{p}}{ }^{2}=0.146\right)$.

Heart rate variability. The effect of drinking water on heart rate variability was considered using RMANOVA where change scores (end of the morning minus baseline) from each day were entered (Table 1; Fig. 2). When participants consumed water they had a larger increase in their average R-R interval, that is they had a lower heart rate $\left(\mathrm{F}(1,11)=5.766, \mathrm{p}<0.03, \eta_{\mathrm{p}}{ }^{2}=0.344\right)$. In addition, when participants had drunk water they had higher heart rate variability as shown by a larger standard deviation of the R-R interval $(\mathrm{F}(1,11)=9.120$, $\mathrm{p}<0.01, \eta_{\mathrm{p}}^{2}=0.395$ ), and a larger root mean squared of standard deviation of R-R interval (a measure of vagal activity) $\left(\mathrm{F}(1,11)=6.128, \mathrm{p}<0.03, \eta_{\mathrm{p}}^{2}=0.357\right)$.

Cognitive performance in the scanner. Data were analysed using RMANOVA with condition (Water, No water) as a repeated measures factor. Main effects are shown in Table 2. The number of non-responses $\left(\mathrm{F}(1,11)=0.277, \mathrm{p}=0.609, \eta_{\mathrm{p}}{ }^{2}=0.020\right)$, reaction times $\left(\mathrm{F}(1,11)=1.884, \mathrm{p}=0.197, \eta_{\mathrm{p}}{ }^{2}=0.146\right)$ and accuracy $\left(\mathrm{F}(1,11)=0.549, \mathrm{p}=0.474, \eta_{\mathrm{p}}^{2}=0.048\right)$ did not depend on whether participants had drunk (Table 2).

Subjective ratings. The subjective responses taken inside and outside the scanner were analysed using RMANOVA with condition (Water, No water) as a repeated measures factor. There were no effects of drinking on ratings of anxiety $\left(\mathrm{F}(1,11)=0.693, \mathrm{p}=0.427, \eta_{\mathrm{p}}{ }^{2}=0.025\right)$, happiness $\left(\mathrm{F}(1,11)=1.875, \mathrm{p}=0.204, \eta_{\mathrm{p}}{ }^{2}=0.172\right)$, energy $\left(\mathrm{F}(1,11)=2.047, \mathrm{p}=0.186, \eta_{\mathrm{p}}{ }^{2}=0.185\right)$ or difficulty $\left(\mathrm{F}(1,11)=0.123, \mathrm{p}=0.733, \eta_{\mathrm{p}}{ }^{2}=0.011\right)$ (Table 2) while participants were in the scanner. However, before entering the scanner (whilst still in the 30 degree environment) participants were significantly less composed when they did not drink water; an effect prevented by drinking $\left(\mathrm{F}(1,11)=4.978, \mathrm{p}<0.047, \eta_{\mathrm{p}}{ }^{2}=0.312\right)$. Participants also tended to be less depressed $(\mathrm{F}(1,11)=4.211$, $\left.\mathrm{p}<0.065, \eta_{\mathrm{p}}{ }^{2}=0.277\right)$, and more energetic $\left(\mathrm{F}(1,11)=2.906, \mathrm{p}<0.116, \eta_{\mathrm{p}}{ }^{2}=0.209\right)$ if they drunk water but these effects did not reach significance.

Brain functioning. fMRI results. A comparison of activation during the arithmetic task in the water compared to the dehydration condition revealed several clusters that achieved statistical significance. A large cluster was centred on the ventral cingulate gyrus (Talairach co-ordinate: 4, 14, -6 ; Fig. 4), that extended into both the hypothalamus and striatal brain regions. Specifically, striatal activity was observed in the bilateral caudate nucleus and putamen. The same cluster also spread inferiorly to right amygdala. To the anterior aspect the activity spread into the medial orbito-frontal cortex. A second small cluster was observed in the dorsal cingulate gyrus (Talairach co-ordinate: 9, 23, 20). The final cluster of activity was seen in left post-central gyrus ('PoCG', Talairach 
RR

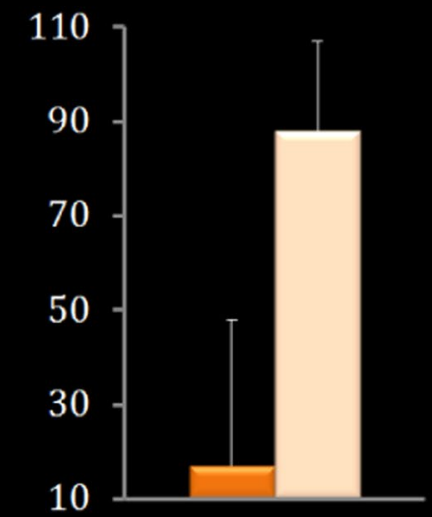

no water Water

RR

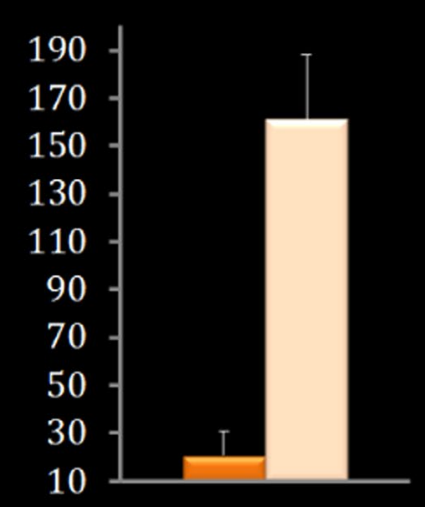

\# No water

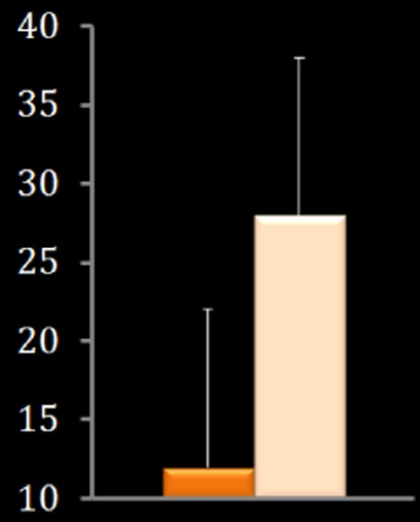

40

m No water $\mathbf{m}$ Water

SDRR

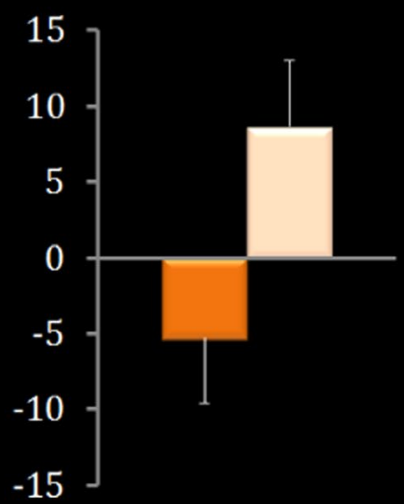

n No water $\mathbf{m a t e r}$
RMSSD

40

35

30

25

20

15

10

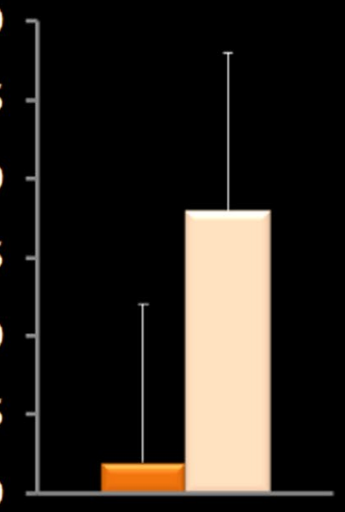

[ No water $\mathbf{W}$ Water

RMSSD

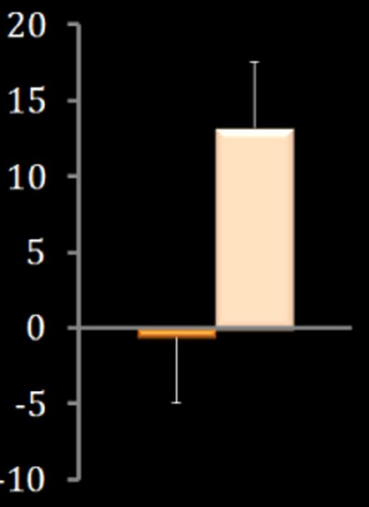

n No water $\boldsymbol{\square}$ Water

Figure 2. The effect of drinking water on the average length of the RR interval, SDNN and RMSSD. Data are the changes (end of the morning minus baseline) for each index compared across the two conditions. Top panel: Study 1 . When participants were hypo-hydrated they had a lower R-R (i.e a higher HR) $(\mathrm{p}<0.03)$, lower SDRR (i.e a lower HRV) $(\mathrm{p}<0.01)$ and reduced parasympathetic activity (i.e. lower RMSSD) $(\mathrm{p}<0.03)$. Bottom panel: Study 2. If water was consumed participants had an increase in their average $R-R$ interval $(p<0.001)$, a larger standard SDNN $(\mathrm{p}<0.027)$, and a larger RMSSD $(\mathrm{p}<0.031)$. RR, interbeat interval, SDNN, standard deviation of normal to normal R-R interval, RMSSD, root mean square of the standard deviation.

co-ordinate: $-36,-19,-35$; Fig. 3 ) and extended superior and anterior into the superior parietal cortex (Figs 3 , 4 and 6). There were no significant areas of activation for the reverse contrast of dehydration $>$ water.

Cerebral blood flow. A voxel wise paired contrast of the perfusion values, obtained via the ASL scans, did not reveal any significant differences in resting perfusion for either the contrast of dehydration vs. water, or water vs. dehydration (all $p$ 's $>0,05$, uncorrected for multiple comparisons).

Association between differences in brain activity, HRV and mood. Peaks of activity occurred on the ventral cingulate gyrus (vCG), the border of the medial orbitofrontal cortex (mOFC) and in the left post-central gyrus (PoCG). To investigate the possible mechanisms behind the observed differences in brain (see above), the difference in brain activity between when participants had, or had not consumed water, was correlated (Pearson's r) with difference scores (no water minus water) for heart rate variability and mood. Results are displayed in Table 3. Heart rate variability correlated significantly with activity in the mOFC $(r=0.78, \mathrm{p}<0.005)$ and vCG areas $(\mathrm{r}=0.77$, $\mathrm{p}<0.05$; Fig. 4); in both instances a smaller increase in R-R interval (i.e a higher heart rate) was associated with lower activity brain activity when no water had been drunk. Similar effects were found for the HRV indices; both the SDNN and RMSSD correlated significantly with activity in the mOFC $(r=0.42, \mathrm{p}<0.05$ for SDNN and $r=0.48, \mathrm{p}<0.05$ for RMSSD) and $\mathrm{vCG}(r=58, \mathrm{p}<0.05$ for mOFC and $r=0.52, \mathrm{p}<0.05$ for vCG) regions, indicating that the hypo-hydration-associated decline in activity may reflect changes in cardiac vagal activity. Overall 


\begin{tabular}{|c|c|c|c|c|}
\hline & \multicolumn{2}{|l|}{ Study 1} & \multicolumn{2}{|l|}{ Study 2} \\
\hline & No Water & Water & No Water & Water \\
\hline $\mathrm{N}^{\circ}$ of non-responses & $20.08(7.50)$ & $16.91(3.21)$ & - & - \\
\hline Reaction time (RT in s) & $0.611(0.02)$ & $0.627(0.01)$ & - & - \\
\hline Accuracy $\left(\mathrm{N}^{\circ}\right.$ correct $)$ & $200.08(9.36)$ & $206.83(3.93)$ & - & - \\
\hline Perceived difficulty (VAS) & $181.16(12.0)$ & $175.00(12.0)$ & - & - \\
\hline Anxiety (VAS) & $103.50(14.6)$ & $90.20(17.78)$ & - & - \\
\hline Happiness (VAS) & $148.60(7.77)$ & $157.50(7.52)$ & - & - \\
\hline Energy levels (VAS) & $112.30(9.69)$ & $132.80(13.72)$ & - & - \\
\hline$\Delta$ Composed (VAS) (outside the scanner) & $-9.50(2.95)^{*}$ & $-0.75(3.26)^{*}$ & $-16.39(5.32)^{*}$ & $-0.07(5.32)^{*}$ \\
\hline$\Delta$ Energetic (VAS) (outside the scanner) & $-14.33(5.94)$ & $-2.33(5.20)$ & $-16.03(4.59)$ & $-16.50(4.59)$ \\
\hline$\Delta$ Depressed (VAS) (outside the scanner) & $-7.91(3.97)$ & $0.41(4.23)$ & $-12.12(3.56)$ & $-7.85(3.56)$ \\
\hline
\end{tabular}

Table 2. Effects of drinking compared with not drinking on performance, perceived difficulty and mood (inside and outside the scanner) in study 1 and study 2. Data are mean (s.e.). VAS, Visual Analogue Scale. *Water vs. No water significant at $\mathrm{p}<0.05$.
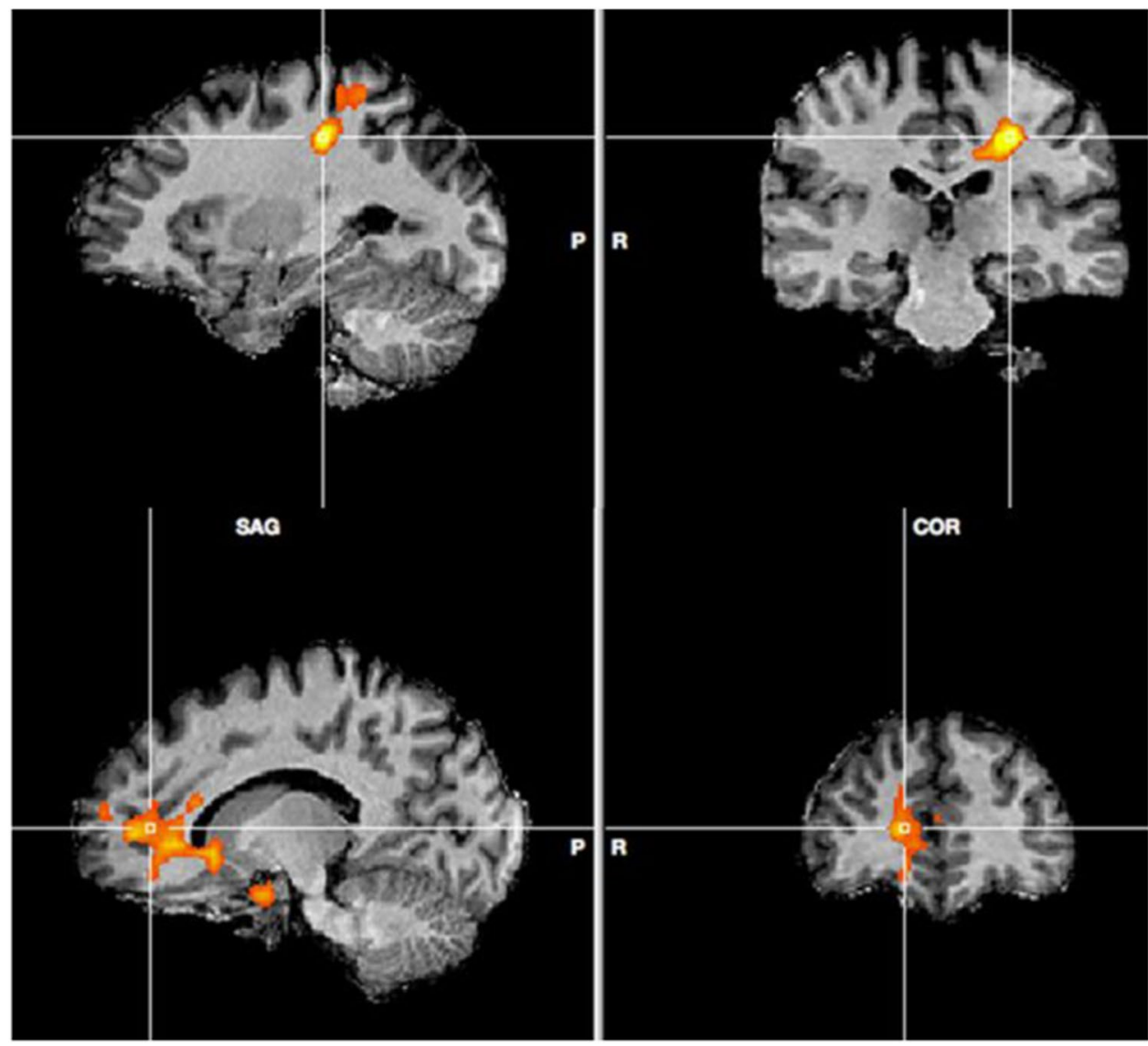

Figure 3. Peak activity in PoCG (top) and mOFC (bottom) water >hypo-hydration.

these findings suggested that the decline in brain activation that was observed when participants didn't drink reflected autonomic responses to the low-level physiological challenge of hypo-hydration.

To determine whether the observed changes in autonomic and brain functioning have consequences for affect these differences were correlated (Pearson's $r$ ) with ratings of perceived difficulty and mood. (Table 4). The average length of the R-R interval correlated with both anxiety $(r=-0.61, \mathrm{p}<0.03)$ and perceived difficulty $(r=-0.45$, $\mathrm{p}=0.06$ ); a lower RR interval (i.e higher heart rate) in the no water condition was related to greater anxiety and more perceived effort (Fig. 4). Similar associations were found between the subjective ratings and activity in the mOFC $(r=-0.72, p<0.01$ for anxiety; $r=-0.60, p<0.02$ for difficulty) and between the subjective ratings and activity in the vCG $(r=-0.77, p<0.007$ for anxiety; $r=-0.75, p<0.004$ for difficulty); in each case lower activity was associated with more anxiety and greater difficulty. Outside the scanner, composure again correlated with the average length of the RR interval $(r=0.47, p<0.05)$, SD of the $R R$ interval $(r=0.44, p<0.05)$, and a depressed mood correlated with activity in the $\mathrm{vCG}(\mathrm{r}=0.75, \mathrm{p}<0.01)$. 

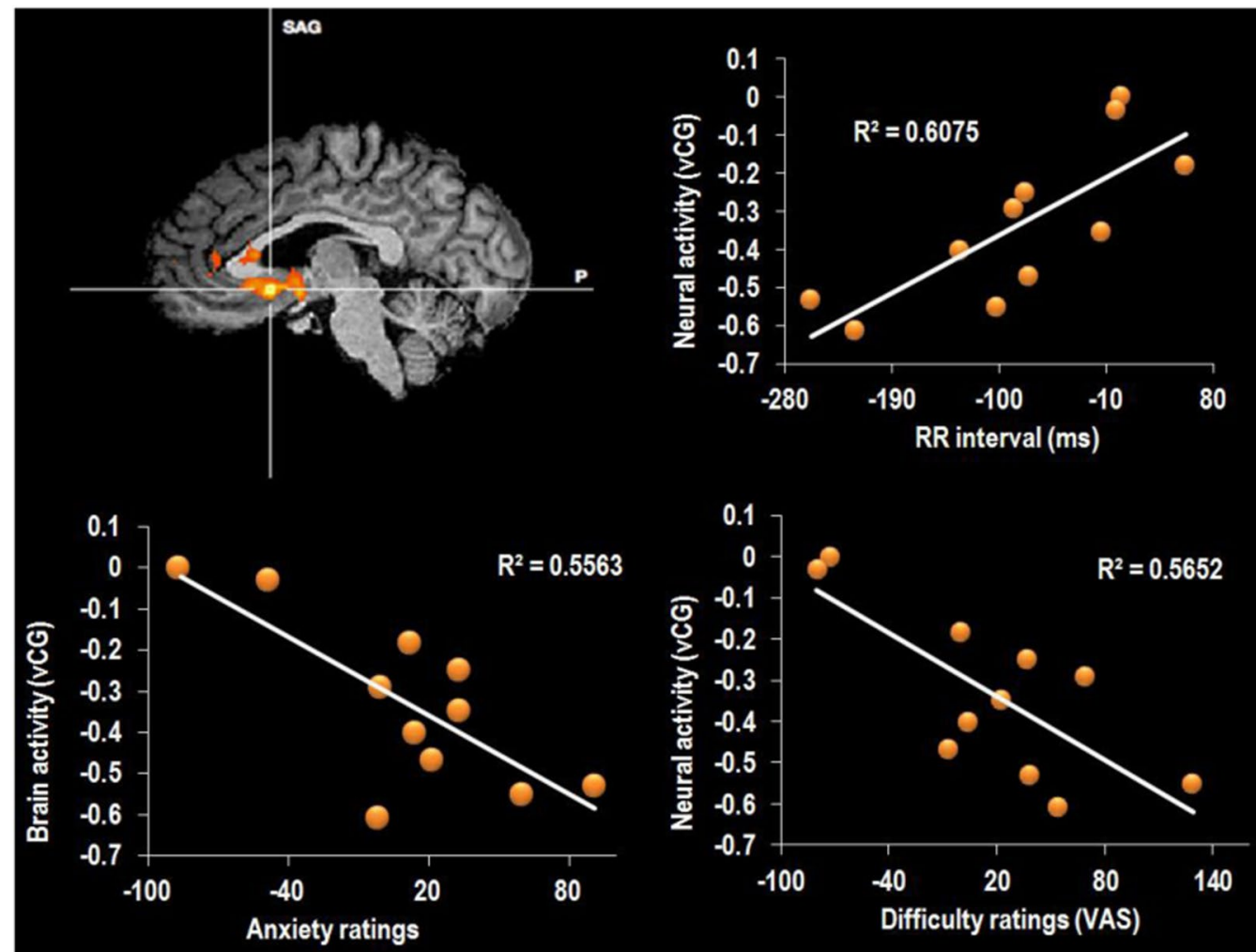

Figure 4. (A) Peak activity in vCG water >hypo-hydration. Correlation between neuronal activity in the vCG and (B). RR interval (C). anxiety and (D). difficulty ratings. Data are Pearson's $r$ coefficient for difference scores (no water - water).

\section{Water / No water}

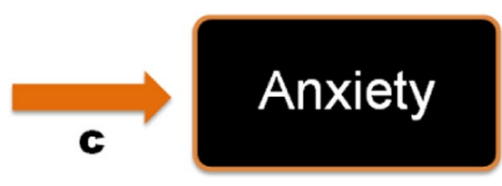

\section{SDNN}

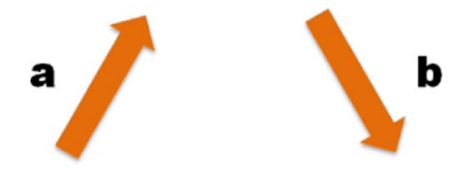

\section{Water / No water}

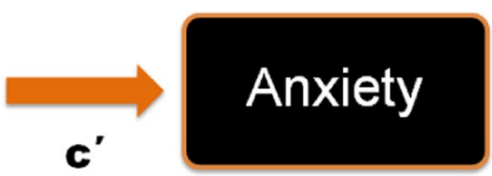

Figure 5. Schematic illustration of the mediation analysis used in study 2 . The total effect of water on rating of anxiety was significant $(B=16.321$, LLCI 3.729, ULCI 28.913). The direct effect (when the influence of SDNN was considered) was not significant $(B=12.763$, LLCI -0.257 , ULCI 25.784). When water was not consumed participants were more anxious $(\mathrm{F}(1,54)=4.706, \mathrm{p}<0.034)$. A decrease in HRV (SDRR) was associated with an increase in anxiety levels $(\mathrm{r}=0.335, \mathrm{p}<0.012)$. The indirect effect Water $\rightarrow \mathrm{SDNN} \rightarrow$ Anxiety was significant $(\mathrm{B}=3.557$, LLCI 0.212, ULCI 8.321). 


\begin{tabular}{|l|l|l|l|}
\hline & mOFC & vCG & PoCG \\
\hline$\Delta$ RR interval & $\mathbf{0 . 7 8 7} * *$ & $\mathbf{0 . 7 7 6} * *$ & 0.145 \\
\hline$\Delta$ SD of R-R interval & $\mathbf{0 . 4 2 8}^{*}$ & $\mathbf{0 . 5 8 0} * *$ & 0.027 \\
\hline$\Delta$ RMSSD & $\mathbf{0 . 4 8 6}^{*}$ & $\mathbf{0 . 5 2 9} *$ & 0.350 \\
\hline
\end{tabular}

Table 3. Correlations between differences in brain activation and differences in heart rate variability. Data are Pearson's correlation coefficient $(\mathrm{r}) \Delta$ Difference, ${ }^{*} \mathrm{p}<<0.005,{ }^{*} \mathrm{p}<0.05, \mathrm{RR}$, interbeat interval, SDNN, standard deviation of normal to normal R-R interval, RMSSD, root mean square of the standard deviation, mOFC, medial orbitofrontal cortex, PoCG, post-central gyrus, vCG, ventral cingulate gyrus.

\begin{tabular}{|c|c|c|c|c|c|c|c|}
\hline & $\begin{array}{l}\text { Perceived } \\
\text { difficulty }\end{array}$ & Happy & Energetic & Anxious & $\begin{array}{l}\text { Depressed (outside } \\
\text { of the scanner) }\end{array}$ & $\begin{array}{l}\text { Energetic (outside } \\
\text { of the scanner) }\end{array}$ & $\begin{array}{l}\text { Composed (outside } \\
\text { of the scanner) }\end{array}$ \\
\hline$\Delta \mathrm{RR}$ interval & $-0.456 *$ & 0.086 & 0.145 & $-0.613 *$ & 0.336 & -0.179 & $0.475^{*}$ \\
\hline$\Delta \mathrm{SD}$ of $\mathrm{R}-\mathrm{R}$ interval & $-0.540 *$ & -0.068 & -0.133 & $-0.746 *$ & $0.650 *$ & -0.041 & $0.445^{*}$ \\
\hline$\Delta$ RMSSD & -0.298 & 0.058 & -0.195 & -0.259 & 0.382 & 0.433 & -0.129 \\
\hline $\mathrm{mOFC}$ & $-0.601 *$ & 0.278 & 0.247 & $-0.722 *$ & 0.393 & -0.101 & 0.158 \\
\hline vCG & $-0.754^{*}$ & -0.228 & -0.296 & $-0.774 *$ & 0.752* & -0.032 & 0.114 \\
\hline PoCG & -0.026 & -0.036 & -0.034 & -0.319 & \begin{tabular}{|l|l|}
0.007 \\
\end{tabular} & 0.471 & 0.103 \\
\hline
\end{tabular}

Table 4. Correlations between differences in brain activation, hydration parameters and performance and difficulty ratings in the scanner. $* p<0.05 \mathrm{RR}$, interbeat interval, SDNN, standard deviation of normal to normal R-R interval, RMSSD, root mean square of the standard deviation, mOFC, medial orbitofrontal cortex, PoCG, post-central gyrus, vCG, ventral cingulate gyrus.

\section{Results: Study 2}

Given the small samples size invariably associated with studies involving neuroimaging a second behavioral study was conducted to replicate and extend the main findings from study 1 . Specifically it was hypothesized that declines in HRV would mediate the effect of hypo-hydration on mood.

Verification of the hydration paradigm. The effect of drinking on percentage total weight loss, perspiration rate and changes in osmolality and thirst were compared across the two conditions using ANOVA. As observed in study 1, participants who did not drink water lost more weight both before $(\mathrm{F}(1,54)=15.963$, $\left.\mathrm{p}<0.0001, \eta_{\mathrm{p}}{ }^{2}=0.227\right)$ and after $\left(\mathrm{F}(1,54)=4.705, \mathrm{p}<0.03, \eta_{\mathrm{p}}{ }^{2}=0.087\right)$ urination; losing an average of $0.55 \%$ of their body mass. In addition, those who didn't drink had a significant increase in urine osmolality $\left(\mathrm{F}(1,54)=6.994, \mathrm{p}<0.011, \eta_{\mathrm{p}}{ }^{2}=0.117\right)$, although the effect of thirst was not significant $(\mathrm{F}(1,54)=1.885$, $\left.\mathrm{p}<0.175, \eta_{\mathrm{p}}^{2}=0.034\right)$. Thus in both studies there was evidence that the current paradigm is a valid way of manipulating small changes in hydration without exposing participants to physical activity (Table 1).

Heart rate variability. The effect of drinking water on heart rate variability was considered by comparing change scores (end of the morning minus baseline) using ANOVA (Table 1; Fig. 2), the findings in study 2 were similar those in study 1 . If water was consumed participants had an increase in their average R-R inter$\operatorname{val}\left(\mathrm{F}(1,54)=13.023, \mathrm{p}<0.001, \eta_{\mathrm{p}}{ }^{2}=0.194\right)$, a larger standard deviation of the R-R interval $(\mathrm{F}(1,54)=5.187$, $\left.\mathrm{p}<0.027, \eta_{\mathrm{p}}{ }^{2}=0.088\right)$, and a larger root mean squared of standard deviation of $\mathrm{R}-\mathrm{R}$ interval $(\mathrm{F}(1,54)=4.873$, $\left.\mathrm{p}<0.031, \eta_{\mathrm{p}}{ }^{2}=0.083\right)$. Therefore, the studies provided consistent evidence that minor hypo-hydration results in an increased heart rate and decreased heart rate variability.

Subjective ratings. Data were analysed using ANOVA where change scores were compared across conditions. When anxiety was considered, participants who did not drink water became significantly more anxious $\left(\mathrm{F}(1,54)=4.706, \mathrm{p}<0.034, \eta_{\mathrm{p}}{ }^{2}=0.080\right)$. However, the effect was not significant when ratings of depression $\left(\mathrm{F}(1,54)=0.734, \mathrm{p}<0.395, \eta_{\mathrm{p}}{ }^{2}=0.013\right)$ and energy $\left(\mathrm{F}(1,54)=0.152, \mathrm{p}<0.698, \eta_{\mathrm{p}}{ }^{2}=0.003\right)$ were considered (Table 2).

Association between HRV and mood. A decrease in average R-R interval was associated with an increase in rating of depression $(\mathrm{r}=0.276, \mathrm{p}<0.040)$, although no association was observed with ratings of anxiety $(\mathrm{r}=0.141, \mathrm{p}<0.301)$ or energy levels $(\mathrm{r}=0.008, \mathrm{p}<0.955)$. A decrease in SDRR was associated with an increase in rating of depression $(\mathrm{r}=0.315, \mathrm{p}<0.018)$ and an increase in anxiety levels $(\mathrm{r}=0.335, \mathrm{p}<0.012)$, but again no association with observed with reported energy $(\mathrm{r}=0.104, \mathrm{p}<0.445)$. RMSSD was not associated with any of the mood measures (anxiety; $\mathrm{r}=0.095, \mathrm{p}<0.488$, depression; $\mathrm{r}=0.215, \mathrm{p}<0.111$, energy levels; $\mathrm{r}=-0.195$, $\mathrm{p}<0.151)$.

To confirm whether changes in HRV mediate the effect of hypo-hydration on mood a mediator analysis was conducted using Hayes PROCESS model 4 (Fig. 5). As anxiety was the only mood measure to show a significant main effect of drinking water this was the dependent variable $(\mathrm{Y})$. Water consumption was the dichotomous independent variable $(\mathrm{X})$, and SDNN was the mediator $(\mathrm{M})$. As reported previously, the total effect of water on rating of anxiety was significant $(B=16.321$, LLCI 3.729, ULCI 28.913), however, the direct effect (when the influence of SDNN was considered) was not significant $(B=12.763$, LLCI -0.257 , ULCI 25.784). The indirect 

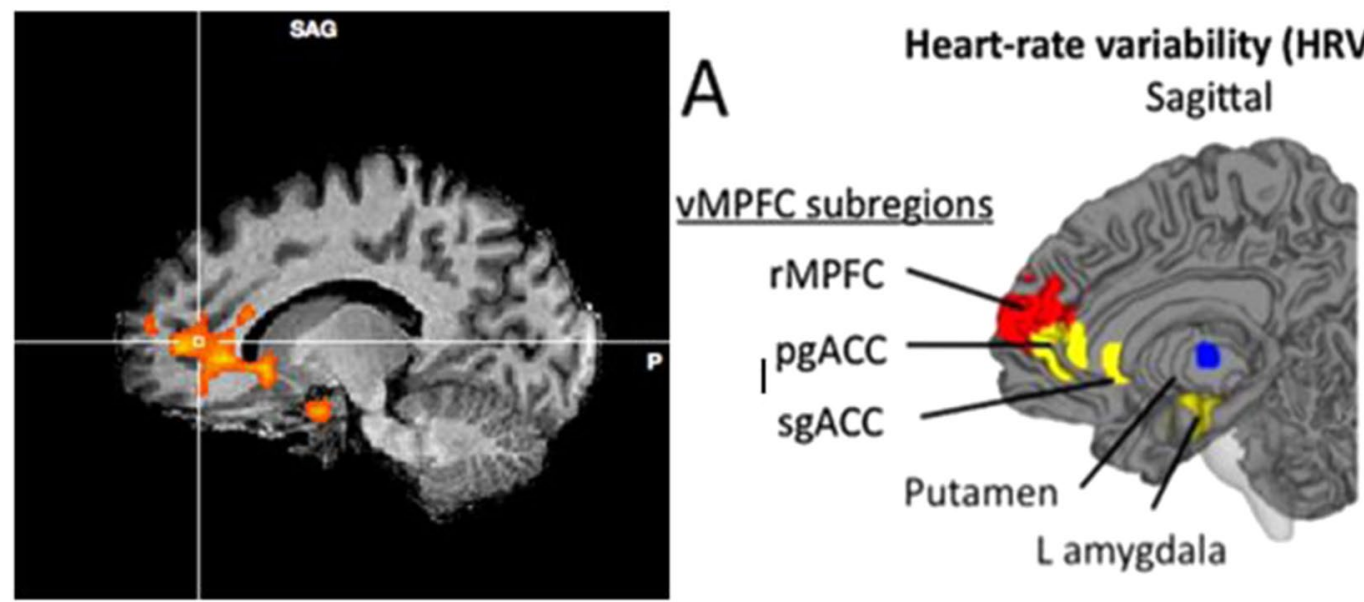

Figure 6. Map showing the brain areas associated with autonomic modulation. Left data from present study. Right areas controlling HRV reproduced from Thayer et al. (2012) with permission.

effect Water $\rightarrow$ SDNN $\rightarrow$ Anxiety was significant $(B=3.557$, LLCI 0.212 , ULCI 8.321). This finding suggested that hypo-hydration associated increases in anxiety levels are fully mediated by autonomic adaptations as indexed by a reduction in HRV.

\section{Discussion}

In two studies it is reported that hypo-hydration adversely influenced mood and brain functioning. In study 1 , as a result of not drinking water during the experimental protocol, task related activity in the autonomic network of the brain was reduced; specifically activity in the orbito-frontal cortex, ventral cingulate gyrus, dorsal cingulate cortex, hypothalamus, amygdala, right striatum, post-central gyrus and superior parietal cortex was affected. When participants were hypo-hydrated they had a higher HR and lower HRV; effects that correlated with peak activation in the vCG and mOFC. Importantly, these effects were associated with a decline in mood. The deleterious effect of hypo-hydration on HRV was replicated in study 2 where this variable was found to mediate the influence of not drinking water on mood. These observations are consistent with the hypothesis that hypo-hydration elicits a change in autonomic regulatory activity and brain function, with potential adverse consequences for aspects of mood.

In the context of exercise severe hypo-hydration ( $>2 \%$ loss of body weight) elevates $\mathrm{HR}^{1}$ but there has been little study of the effect of everyday fluctuations in hydration status on cardiac functioning. The present studies found that in participants at rest, hypo-hydration resulted in a shorter average R-R interval i.e a higher HR (on average 5.8 beats per minute higher) and lower HRV (Fig. 2). Interestingly, limited evidence suggests that drinking water, even in the absence of hypo-hydration, may modulate the cardiac vagal response; 20 and 25 min after drinking $500 \mathrm{ml}$ of water heart rate fell from 67 to $60 \mathrm{bpm}$ and RMSSD increased by $13 \mathrm{~ms}^{15}$. This latter finding is important because it might help explain how acute water supplementation can benefit mood, irrespective of hydration status ${ }^{30}$.

Areas of the brain mediating sympathetic and parasympathetic control of the ANS include regions of the limbic forebrain such as the subgenual and pregenual cingulate, amygdala/ventral striatum and medial prefrontal cortex ${ }^{20}$ : as can be seen in Fig. 6, changes in many of the same regions were associated with hypo-hydration. Mathews et al. ${ }^{31}$ studied the association between activity in the VCG and peak high frequency power (a HRV index of vagal activity) during a Stroop task; they found a positive association such that greater activity in the vCG was associated with a higher vagal tone. Similarly, in the present study, having higher activity in the vCG and mOFC was associated with greater parasympathetic activity (indexed by the average RR interval, SDRR and RMSSD). These findings indicated that the hydration related differences in brain activity in these regions may be the result of hydration induced autonomic modulation. Interestingly, drinking water both ameliorated the task related decline in brain activity, as indicated by fMRI, and increased HRV. A novel hypothesis is that hypo-hydration induces increased cardiovascular strain, which in turn necessitates a decline in activity in the autonomic network of the brain, effects that could compromise cognitive and affective processing.

Even in a healthy population lower HRV is associated with poorer mood and cognition and higher perceived stress $^{22}$. Similarly, study 1 found that a higher heart rate and lower HRV were associated with increases in anxiety and perceived effort during scanning; these effects were also associated with having a greater deactivation in the mOFC and vCG. In addition, the association between lower HRV and increased anxiety was replicated in study 2. Hypo-activation of the anterior cingulate and frontal cortices is common in a range of anxiety disorders ${ }^{32}$ and during induced anxiety ${ }^{33}$. In addition, interventions known to reduce anxiety increase activity in these regions ${ }^{34}$; it is therefore plausible that the benefits of drinking water reflect similar mechanisms. These observations are important as they may have clinical implications for populations characterized by raised anxiety levels.

Notably, in study 1 , mood monitored while participants were in the scanner did not differ according to whether or not they consumed water (Table 2), however, before they left the $30^{\circ} \mathrm{C}$ environment (see methods 
section) there were between condition differences in anxiety; an effect subsequently replicated in study 2 . Ambient temperatures in excess of $27^{\circ} \mathrm{C}$ exacerbate the effects of dehydration, induce peripheral vasodilatation and result in relative hypovolemia, increasing cardiac strain ${ }^{1}$. Thus, autonomic adaptations that mediate the effects of hydration are likely to be enhanced by a warm environment. It is plausible that by the time participants entered the scanner (on average $22^{\circ} \mathrm{C}$ ) the exacerbating effect of heat would have been diminished. Supporting this view, Farrell et al. ${ }^{35}$ recently reported that regions of the central autonomic network were activated in response to both thermogenic and psychogenic sweating, in particular the anterior cingulate cortex, lentiform nuclei and parietal cortex. In the present study perspiration rate correlated significantly with activity in all three regions of difference; the mOFC, vCG and PoCG (Table S1). If such regions are also involved in hypo-hydration associated cognitive and affective deficits, this might help to explain the effects of the interaction between thermoregulation and hypo-hydration on cognition and mood. Future research might consider replicating these findings using a water deprivation paradigm that doesn't involve exposure to increased ambient temperatures.

The present findings support the hypothesis that even a relatively minor decline in hydration necessitates a counter-regulatory autonomic response which may have affective consequences. However, an alternative hypothesis might be that, rather than altering neural activity, hypo-hydration reduces cerebrovascular coupling. Given that brain functional imaging methods, such as fMRI, are sensitive to changes in cerebral blood flow $(\mathrm{CBF})$ it is plausible that the observed differences in regional BOLD activity might reflect differences in CBF. Hypo-hydration of $1.3 \%$ reduced mean middle cerebral artery blood flow velocity during a cold pressor test, suggesting that the cerebrovascular response to acute stressful stimuli may be altered by hydration status ${ }^{36}$. However, the present study found no effects of hydration on global or regional blood flow using resting state ASL; nonetheless, it remains possible that dynamic cerebrovascular reactivity, rather than resting state auto-regulation, may have been compromised under the present conditions. This remains an important consideration for future research that examines the effects of hydration on brain functioning using imaging.

The limitations of the present study should be considered. Firstly, it is unclear whether hypohydration related changes in anxiety and perceived effort are driving the associated differences in HRV and brain activity, or vice versa. Further research might consider the potential influence of individual differences in afferent baroreceptor activity and interoceptive abilities which has been shown to modulate the processing of emotional informa$\operatorname{tion}^{37,38}$, and may be increased following water consumption. A factor that could limit the generalizability of the results is the small sample sizes. Although we were able to replicate findings, the possibility exists that the studies were underpowered to detect some smaller differences in mood (e.g. energy levels/depression). In addition, the sample comprised only young university students and future research may consider different populations who may be more susceptible to the effects of hypohydration; for example children and older adults. Finally, although the majority of studies of HRV have not measured respiration it is potentially a confounding variable. Although there is no reason to believe that respiration varied systematically, such that it would have biased the present findings, it is a question to be addressed in future studies.

In conclusion, the present study reports that when participants consumed water, compared with when they were mildly hypohydrated ( $0.6 \%$ loss in body weight) they had improved mood as indicated by VAS ratings, and increased neural activity as indicated by fMRI when faced with a demanding task. For the first time we have highlighted important regulatory neural mechanisms that may account for the psychological benefits of maintaining hydration status. When water was not consumed more weight was lost, osmolality increased and HRV decreased; effects that predicted a larger task related deactivation in the autonomic network of the brain. Only recently have the interactions between bodily reactions and affective processes begun to be elucidated and such phenomena are still often treated as confounding factors. Indices of ANS function have been used as objective measures of affective states and these responses have been treated as epiphenomena, rather than intrinsic to the cognitive or emotional process. As evidenced here, it is possible that changes in ANS activity have consequences for mood. As such hypo-hydration to the extent that it influences ANS activity per se may have negative consequences for brain functioning and mood.

Given the prevalence of voluntary hypo-hydration these findings have important implications for vulnerable cross-sections of the population. Furthermore, the psychological benefits of drinking may have important implications for populations, such as older adults and children ${ }^{39}$ who are at a significant risk of dehydration. The fact that mild changes in hydration affected cardiovascular functioning is also a concern; the HR of participants was on average 5.8 beats per minute lower when they had consumed water. Given that there is a progressive increase in the risk of heart-disease as resting heart rate increases ${ }^{40}$, and hyper-osmolarity predicted a 4.3 -fold increased risk of cardiovascular mortality ${ }^{41}$, maintaining adequate hydration may be an important factor for maintenance of cardiovascular health. In general, repeated low level physiological challenges such as hypo-hydration may overtime increase allostatic load, predisposing to possible negative health outcomes.

\section{Methods}

Sample characteristics (Study 1). The sample size was based on the expected power for a hypothesized within participants effect. Total sample size was calculated using $G^{*}$ power based on the following parameters: one group, two within subject levels (Water/No water) with an expected correlation of $0.6, \alpha=0.05$, a two-tailed test, and $80 \%$ power to detect a medium to large sized effect (Cohen's $\mathrm{f} 2=0.4$ ). This gave an estimated $\mathrm{N}$ of 12 which is in line with previous studies that have examined the neural effects of hydration e.g. ${ }^{26}$.

Twelve healthy young males (average age 23.3 years (19-34), average BMI 25.4 (19-34)), gave their written informed consent after being screened for MRI safety. Participants were excluded if they had any health complaint that would affect cardiovascular functioning such as diabetes or hypertension. Similarly, anyone with a neuropsychological illness was also excluded as were those taking medication. All participants were right handed, non-smokers; before the start of the study they were asked to refrain from drinking alcohol for at least 24 hours and told to fast and avoid any beverages for at least twelve hours. 




Figure 7. Arithmetic task and Visual analogue scale example stimuli. The crosses are fixation points when otherwise the screen is blank. At the third image the participant indicated whether the right or left number was correct by pushing a button.

Sample characteristics (Study 2). The sample size was based on the expected power for a hypothesized between participants effect. Total sample size was calculated using $\mathrm{G}^{*}$ power based on the following parameters that were deduced from study 1: two groups (Water/No water), $\alpha=0.05$, a two-tailed test, and $80 \%$ power to detect a medium to large sized effect (Cohen's $\mathrm{f}=0.4$ ). This gave an estimated $\mathrm{N}$ of 52 .

Twenty-five males and thirty-one females (average age 21.0 years (18-28), average BMI 23.3 (17-35)), gave their written informed consent and were randomly allocated to either the water or no water condition (Water 15F, $13 \mathrm{M}$, No water $16 \mathrm{~F}, 12 \mathrm{M}$ ). As it has been suggested that gender may be a determining factor in the correlation between hypo-hydration and its psychological consequences ${ }^{2}$, this factor was initially considered. Preliminary analysis found no significant interactions between gender and water consumption for any of the dependent variables (all $\mathrm{p}>0.1$ ). Exclusion criteria and pre-study instructions were the same as for study 1 .

Procedure (Study 1). On two occasions participants attended the laboratory that was heated to 30 degrees. Using a repeated measures design, in a randomly derived order, they received either two $150 \mathrm{ml}$ glasses of water or nothing (Fig. 1). Upon arriving at the laboratory, participants were asked to provide a urine sample and were asked to completely empty their bladder, following which they were weighed and their body temperature measured. They were then fitted with a RS800 Polar heart rate monitor electrode transmitter belt (T61) and a Polar RS800 HR monitor (Polar Electro, Kempele, Finland) that was used to collect interbeat interval measurements at a sampling rate of $1000 \mathrm{~Hz}$, while participants rested in a seated position. This instrument has been previously validated for the accurate measurement of R-R intervals and for analysing Heart Rate Variability $(\mathrm{HRV})^{42}$. Participants were then provided with a standard breakfast consisting of $50 \mathrm{~g}$ of Quaker Oat So Simple Original Porridge ( $187 \mathrm{kcal}, 2.9 \mathrm{~g}$ fat, $10.6 \mathrm{~g}$ sugar, $0.11 \mathrm{~g}$ salt) plus $150 \mathrm{ml}$ of either decaffeinated tea or coffee. After breakfast participants were weighted again, and rated their mood and thirst. After this they were allowed to rest while either watching TV or reading. Three hours later participants were asked to provide another urine sample and again had their HRV, body temperature and weight measured, and rated their mood and thirst. Finally, they were escorted to the MRI laboratory for the scanning procedure. The procedure was approved by Swansea University ethics committee (ref: 01.01.2015.1) and carried out in accordance with the principles laid down by the declaration of Helsinki 2013. ClinicalTrials.gov Identifier: NCT03525470 (15/05/18).

Procedure (Study 2). The procedure for study 2 was identical with the exception that it employed a between participants rather than within subject design. In addition, participants did not complete the fMRI protocol. The procedure was approved by Swansea University ethics committee (ref: 01.03.2018.1) and carried out in accordance with the principles laid down by the declaration of Helsinki 2013. These data were collected as part of a larger trial: ClinicalTrials.gov Identifier: NCT02671149 (02/02/16).

Mood (Study 1 and Study 2). Mood measures were identical across both studies. Participants were asked to report on visual analogue scales how they felt "at this moment" using visual analogue scales with pairs of adjectives at the ends of $100 \mathrm{~mm}$ lines; Composed/Anxious; Elated/Depressed; Energetic/Tired; as described by McNair and Lorr ${ }^{43}$. 
Thirst (Study 1 and Study 2). In both studies, participants were asked to responds to the question "how thirsty are you feeling right now" on a single $100 \mathrm{~mm}$ visual analogue scale anchored by "Not at all" and "Extremely".

Osmolality (Study 1 and Study 2). The osmolality of urine was assessed using an Osmomat 3000 freezing point osmometer (Gonotec GmbH, Berlin, Germany).

Body temperature (Study 1). Body temperature was measured using a TH8 Infrared Ear Thermometer (Radiant Innovation, Taiwan).

Body mass (Study 1 and Study 2). Body mass was measured using an electronic scale (Kern KMS-TM, Kenr and Sohn GmbH, Germany) that, to avoid problems associated with movement, took 50 assessments over a 5 second period and produced an average value. It was sensitive enough to weigh to within 5 grams ( $17 \%$ of an ounce) and could pick up over short periods changes in body mass due to breathing and perspiration. Participants were weighed on arrival, both before and after breakfast, and again at the end, both before and after urination.

Scanning procedure (Study 1). Participants were presented with an arithmetic task, modified for use in the scanner, similar to the Paced Auditory Serial Addition Task: a 'stressful' task that measures calculation ability and is known to elicit an autonomic response ${ }^{44}$. Pairs of two digit numbers appeared on a screen in red and participants were required to mentally add or subtract the numbers. After 2 seconds the screens was removed and a second screen appeared containing a correct and an incorrect answer. Participants were required to press either a left or right button to indicate which answer was correct (Fig. 7). The speed of presentation is designed to be just at the level that it is possible to perform the task although it required mental effort and was 'stressful'. This allowed investigation of areas of the brain associated with working memory but also those associated with emotional arousal. Data analysed from the task were number of missed responses, the number of correct responses and average reaction time in milliseconds (on the trials that participants responded). Three blocks of four minutes were performed. At the end of each block four visual analogue scales appeared and participants were asked to rate, using the left and right key to move a cursor, how difficult they found the proceeding block and how happy, energetic and anxious they felt at that moment. The visual analogue scales were rated on a scale of 1-100.

Received: 8 June 2018; Accepted: 23 October 2019;

Published online: 11 November 2019

\section{References}

1. Sawka, M. N., Cheuvront, S. N. \& Kenefick, R. W. Hypohydration and Human Performance: Impact of Environment and Physiological Mechanisms. Sports Medicine 45, 51-60 (2015).

2. Adan, A. Cognitive performance and dehydration. Journal of the American College of Nutrition 31, 71-78 (2012).

3. Benton, D. Dehydration influences mood and cognition: a plausible hypothesis? Nutrients 3, 555-573 (2011).

4. Benton, D., Jenkins, K. T., Watkins, H. T. \& Young, H. A. Minor degree of hypohydration adversely influences cognition: a mediator analysis. The American Journal of Clinical Nutrition 104, 603-612 (2016).

5. Young, H. A. \& Benton, D. The use of moderated mediated analysis to study the infl uence of hypo-hydration on working memory. Nutricion hospitalaria 33, 71-75 (2016).

6. Watson, P., Whale, A., Mears, S. A., Reyner, L. A. \& Maughan, R. J. Mild hypohydration increases the frequency of driver errors during a prolonged, monotonous driving task. Physiology \& behavior 147, 313-318 (2015).

7. Neave, N. et al. Water ingestion improves subjective alertness, but has no effect on cognitive performance in dehydrated healthy young volunteers. Appetite 37, 255-256 (2001)

8. Rogers, P. J., Kainth, A. \& Smit, H. A drink of water can improve or impair mental performance depending on small differences in thirst. Appetite 36, 57-58 (2001).

9. Hew-Butler, T. \& Panel, T. E. C. Inadequate hydration or normal body fluid homeostasis? American journal of public health 105, e5 (2015).

10. Tanner. The Regulation of Fluid and Electrolyte Balance. In: Rhoades, R. A. and Bell, D. R., ed. Medical Physiology Principles for Clinical Medicine. 3rd ed. Philadelphia: Lippincott Williams and Wilkins. 419-441 (2009).

11. Adams, W. M., Ferraro, E. M., Huggins, R. A. \& Casa, D. J. Influence of body mass loss on changes in heart rate during exercise in the heat: a systematic review. The Journal of Strength \& Conditioning Research 28, 2380-2389 (2014).

12. Trangmar, S. J. et al. Dehydration accelerates reductions in cerebral blood flow during prolonged exercise in the heat without compromising brain metabolism. American Journal of Physiology-Heart and Circulatory Physiology 309, H1598-H1607 (2015).

13. Schroeder, C. et al. Water drinking acutely improves orthostatic tolerance in healthy subjects. Circulation 106, 2806-2811 (2002).

14. Rochette, L. M. \& Patterson, S. M. Hydration status and cardiovascular function: effects of hydration enhancement on cardiovascular function at rest and during psychological stress. International journal of psychophysiology 56, 81-91 (2005).

15. Helen, C., Chowdhary, S., Coote, J. H. \& Townend, J. N. Cardiac vagal response to water ingestion in normal human subjects. Clinical Science 103, 157-162 (2002)

16. Denton, D. et al. Correlation of regional cerebral blood flow and change of plasma sodium concentration during genesis and satiation of thirst. Proceedings of the National Academy of Sciences 96, 2532-2537 (1999).

17. Egan, G. et al. Neural correlates of the emergence of consciousness of thirst. Proceedings of the National Academy of Sciences 100, 15241-15246 (2003).

18. Thayer, J. F. \& Lane, R. D. A model of neurovisceral integration in emotion regulation and dysregulation. Journal of affective disorders 61, 201-216 (2000)

19. Farrell, M. et al. Effect of aging on regional cerebral blood flow responses associated with osmotic thirst and its satiation by water drinking: a PET study. Proceedings of the National Academy of Sciences 105, 382-387 (2008).

20. Thayer, J. F., Åhs, F., Fredrikson, M., Sollers, J. J. \& Wager, T. D. A meta-analysis of heart rate variability and neuroimaging studies: implications for heart rate variability as a marker of stress and health. Neuroscience \& Biobehavioral Reviews 36, 747-756 (2012).

21. Beissner, F., Meissner, K., Bär, K.-J. \& Napadow, V. The autonomic brain: an activation likelihood estimation meta-analysis for central processing of autonomic function. The Journal of Neuroscience 33, 10503-10511 (2013).

22. Young, H. \& Benton, D. We should be using nonlinear indices when relating heart-rate dynamics to cognition and mood. Scientific reports 5 (2015). 
23. Young, H. A., Cousins, A. L., Watkins, H. T. \& Benton, D. Is the link between depressed mood and heart rate variability explained by disinhibited eating and diet? Biological psychology 123, 94-102 (2017).

24. Young, H. A. \& Watkins, H. Eating disinhibition and vagal tone moderate the postprandial response to glycemic load: a randomised controlled trial. Scientific Reports 6, 35740, https://doi.org/10.1038/srep35740 (2016).

25. Hovland, A. et al. The relationships among heart rate variability, executive functions, and clinical variables in patients with panic disorder. International Journal of Psychophysiology 86, 269-275 (2012).

26. Kempton, M. J. et al. Dehydration affects brain structure and function in healthy adolescents. Human brain mapping 32, 71-79 (2011).

27. Tarvainen, M. P., Niskanen, J.-P., Lipponen, J. A., Ranta-Aho, P. O. \& Karjalainen, P. A. Kubios HRV-heart rate variability analysis software. Computer methods and programs in biomedicine 113, 210-220 (2014).

28. Goebel, R. BrainVoyager-past, present, future. Neuroimage 62, 748-756 (2012).

29. Talairach, J. \& Tournoux, P. Co-planar stereotaxic atlas of the human brain. 3-Dimensional proportional system: an approach to cerebral imaging. (1988).

30. Masento, N. A., Golightly, M., Field, D. T., Butler, L. T. \& van Reekum, C. M. Effects of hydration status on cognitive performance and mood. British Journal of Nutrition 111, 1841-1852 (2014).

31. Matthews, S. C., Paulus, M. P., Simmons, A. N., Nelesen, R. A. \& Dimsdale, J. E. Functional subdivisions within anterior cingulate cortex and their relationship to autonomic nervous system function. Neuroimage 22, 1151-1156 (2004).

32. Etkin, A. \& Wager, T. D. Functional neuroimaging of anxiety: a meta-analysis of emotional processing in PTSD, social anxiety disorder, and specific phobia. American Journal of Psychiatry (2007).

33. Straube, T., Schmidt, S., Weiss, T., Mentzel, H.-J. \& Miltner, W. H. Dynamic activation of the anterior cingulate cortex during anticipatory anxiety. Neuroimage 44, 975-981 (2009).

34. Zeidan, F., Martucci, K. T., Kraft, R. A., McHaffie, J. G. \& Coghill, R. C. Neural correlates of mindfulness meditation-related anxiety relief. Social cognitive and affective neuroscience 9, 751-759 (2014).

35. Farrell, M. J., Trevaks, D., Taylor, N. A. \& McAllen, R. M. Brain stem representation of thermal and psychogenic sweating in humans. American Journal of Physiology-Regulatory, Integrative and Comparative Physiology 304, R810-R817 (2013).

36. Perry, B. G., Bear, T. L., Lucas, S. J. \& Mündel, T. Mild dehydration modifies the cerebrovascular response to the cold pressor test. Experimental physiology 101, 135-142 (2016).

37. Gray, M. A. et al. Emotional appraisal is influenced by cardiac afferent information. Emotion 12, 180 (2012).

38. Young, H. A., Gaylor, C. M., de Kerckhove, D., Watkins, H. \& Benton, D. Interoceptive accuracy moderates the response to a glucose load: a test of the predictive coding framework. Proc. R. Soc. B, https://doi.org/10.1098/rspb.2019.0244 (2019).

39. Benton, D. \& Burgess, N. The effect of the consumption of water on the memory and attention of children. Appetite 53, 143-146 (2009).

40. Fox, K. et al. Resting heart rate in cardiovascular disease. Journal of the American College of Cardiology 50, 823-830 (2007).

41. Briongos-Figuero, S. et al. Blood hyperosmolarity as a predictor of mortality in patients after acute coronary syndrome: how dehydration affects the prognosis of our patients. Journal of the American College of Cardiology 61 (2013).

42. Nunan, D. et al. Validity and reliability of short-term heart-rate variability from the Polar S810. Medicine+ Science in Sports + Exercise 41, 243 (2009).

43. Lorr, M., McNair, D. M. \& Fisher, S. Evidence for bipolar mood states. Journal of personality assessment 46, 432-436 (1982).

44. Tanosoto, T. et al. Effects of the Paced Auditory Serial Addition Task (PASAT) with different rates on autonomic nervous system responses and self-reported levels of stress. Journal of oral rehabilitation 42, 378-385 (2015).

\section{Acknowledgements}

The authors would like to thank Heather Watkins and Nadine Garland for their assistance in collecting and inputting data for this study. This research was part funded by PepsiCo Inc. JMF is an employee of PepsiCo Inc. The authors have no other conflicts of interest to declare. The views expressed in this manuscript are those of the authors and do not necessarily reflect the position or policy of PepsiCo Inc. PepsiCo Inc. made no contribution to study 2 .

\section{Author contributions}

H.Y., D.B., S.J. and J.M.F. designed the research. H.Y. and S.J. analysed the data. H.Y., D.B., S.J. and J.M.F. prepared and edited the manuscript. H.Y., A.C. and S.J. conducted the research.

\section{Competing interests}

The authors declare no competing interests.

\section{Additional information \\ Correspondence and requests for materials should be addressed to H.A.Y.}

Reprints and permissions information is available at www.nature.com/reprints.

Publisher's note Springer Nature remains neutral with regard to jurisdictional claims in published maps and institutional affiliations.

Open Access This article is licensed under a Creative Commons Attribution 4.0 International License, which permits use, sharing, adaptation, distribution and reproduction in any medium or format, as long as you give appropriate credit to the original author(s) and the source, provide a link to the Creative Commons license, and indicate if changes were made. The images or other third party material in this article are included in the article's Creative Commons license, unless indicated otherwise in a credit line to the material. If material is not included in the article's Creative Commons license and your intended use is not permitted by statutory regulation or exceeds the permitted use, you will need to obtain permission directly from the copyright holder. To view a copy of this license, visit http://creativecommons.org/licenses/by/4.0/.

(C) The Author(s) 2019 